\title{
Filigrane
}

Écoutes psychothérapiques

\section{Confiance en la créativité de l'être. Entretien avec Pierre Drapeau}

\section{Louise Quintal}

Volume 15, numéro 2, automne 2006

URI : https://id.erudit.org/iderudit/014464ar

DOI : https://doi.org/10.7202/014464ar

Aller au sommaire du numéro

Éditeur(s)

Revue Santé mentale au Québec

ISSN

1192-1412 (imprimé)

1911-4656 (numérique)

Découvrir la revue

Citer ce document

Quintal, L. (2006). Confiance en la créativité de l'être. Entretien avec Pierre

Drapeau. Filigrane, 15(2), 28-50. https://doi.org/10.7202/014464ar d'utilisation que vous pouvez consulter en ligne.

https://apropos.erudit.org/fr/usagers/politique-dutilisation/ 


\title{
Confiance en la créativité de l'être. Entretien avec Pierre Drapeau
}

\author{
louise quintal
}

L. Q. : Le nom de Pierre Drapeau évoque pour la plupart de nos lecteurs le monde des enfants, plus particulièrement l'univers maternel. Puis-je demander à celui qui est devenu au Québec une figure emblématique de la psychanalyse d'enfants de nous parler un peu de son enfance?

P. D. : Raconter son enfance est toujours un peu difficile ; à première vue je ne vois pas ce que ce récit pourrait avoir d'intéressant pour les autres, mais j'accepte d'en dire un mot dans la mesure où ces images du passé nous permettent de comprendre le rôle que mon enfance a pu jouer dans mon devenir. L'enfance n'est pas l'infantile mais certains éléments, sûrement transformés et embellis dans mon souvenir, aident à comprendre par où je suis passé et comment je suis devenu ce que je suis aujourd'hui, comment des éléments dus au hasard se sont transformés en destin pour moi..

Je suis né à l'Isle Verte, dans le Bas du Fleuve, au bord de la mer, face aux grands espaces qui portent beaucoup à rêver. J'ai toujours eu une tendance à rêver. J'ai eu le bonheur de grandir dans un milieu sain, à l'abri des carences et des traumatismes avec un père, médecin de campagne, chef absolument incontesté de la famille, une mère aimante, douce, sensible, sûrement un peu surprotectrice, un frère aîné qui m'impressionnait par sa force et son intelligence et puis deux sœurs plus jeunes, très jolies et gentilles. Somme toute, dans un environnement heureux et calme, mais qui avait tout ce qu'il fallait pour constituer le noyau d'une névrose infantile, afin que de façon mystérieuse l'histoire d'Edipe se répète encore une fois. J'étais un enfant assez sage, timide, avec une vie fantasmatique très développée, qui se sentait souvent inférieur, inhibé même intellectuellement. Un grand déblocage mystérieux s'est produit le jour où je suis parti pour le collège, où je me suis éloigné de ma famille pour poursuivre mon cours classique, qui a duré huit ans. Au collège, j'étais un travailleur acharné et je réussissais très bien. J'étais passionné par l'histoire des civilisations, les débuts de l'homme. J'étais sportif aussi, et le fait d'être lanceur à la balle molle pour l'équipe de ma classe m'a donné confiance en moi ; j'ai aussi remporté des tournois au tennis, sport qu'ensuite j'ai pratiqué toute ma vie.

En classe de philosophie, à la fin de mes études, j'ai été très troublé, interrogé, par ce que m'avait dit un curé névrosé, souffrant de tics, qui avait été en thérapie et avait lu Freud. Il m'avait dit à mon sujet des choses surprenantes. J'avais 
commencé à me poser des questions sur moi-même. Je me souviens que j'allais parfois en retraite volontaire au monastère de St-Benoît du Lac, et c'est là, dans le silence du monastère, que j'ai lu l'Abrégé de psychanalyse; ce fut pour moi une révélation.

L. Q. : Ce fut le point de départ de votre intérêt pour la psychanalyse?

P. D.: Le terrain avait déjà été préparé. Mon père, médecin de campagne, disait toujours que $90 \%$ des problèmes de ses patients étaient d'origine psychologique, ce qui m'impressionnait beaucoup. À ce moment-là, les médicaments et les techniques de guérison n'étaient pas très avancés ; les antibiotiques n'existaient même pas et j'avais toujours l'impression qu'il arrivait à guérir ses patients par son très grand engagement, son très grand dévouement, je dirais même sa tendresse envers eux et surtout par la confiance inouïe qu'il leur inspirait et qui faisait que les choses finalement rentraient dans l'ordre. Plus tard, la lecture de Freud m'a convaincu qu'il avait raison. Je me suis orienté vers la médecine mais avec l'idée bien arrêtée de me spécialiser en psychiatrie. C'est ainsi que tout a commencé.

Pendant les deux dernières années de mon cours de médecine, avant même d'être en psychiatrie, je m'étais engagé comme infirmier à l'hôpital St-MichelArchange. J'ai vécu là au plus près de la psychose, avant l'utilisation de la médication, au temps des camisoles de force, des délires spectaculaires, des traitements au choc insulinique... Les religieuses assuraient mon logement et ma nourriture; le reste du temps j'étudiais et je travaillais. J'ai commencé ensuite mes études en psychiatrie à Québec. Et là, un événement très important pour moi est survenu : $\mathrm{D}^{\mathrm{r}}$ Yvon Gauthier, qui venait de Montréal une fois par mois, a commencé à nous faire des présentations inspirées par la compréhension psychodynamique. C'était un homme simple, aimable, intelligent et qui me fascinait. Je m'organisais toujours pour aller le reconduire à l'aéroport après ses cours afin de pouvoir parler avec lui et c'est lui qui m'a invité à venir à Montréal continuer ma formation en pédopsychiatrie à l'hôpital Sainte-Justine.

L. Q. : Il fut un maître pour vous ?

P. D. : Oui, il fut le premier maître que j'ai croisé sur ma route et qui a marqué mon parcours professionnel. Analyste d'enfants, il dirigeait l'enseignement à l'hôpital Sainte-Justine ; il est devenu plus tard directeur du département de psychiatrie universitaire et a eu une brillante carrière. Pendant longtemps, j'ai travaillé intensément avec lui en consultation auprès des enfants hospitalisés en pédiatrie Yvon avait suivi une formation aux États-Unis et s'inspirait beaucoup des travaux d'Anna Freud et de la psychologie du moi; j'ai beaucoup approfondi cette approche qui m'était excessivement utile dans mon travail en plus de m'apporter une compréhension fondamentale du développement de l'enfant.

En quittant le service des consultations j'ai connu une période de très grand engagement social pendant laquelle j'ai dirigé le département de psychiatrie de Sainte-Justine. Un jour, nous y avons invité le grand psychanalyste français René Diatkine et j'ai eu la chance d'avoir pendant deux semaines un contact excessivement privilégié avec lui. Avec cet homme brillant qui aimait parler tard la nuit, j'ai 
eu de longs échanges qui m'ont fait découvrir un autre aspect de la psychanalyse française, mais j'ai aussi entendu parler d'un nouvelle façon de Klein, de Winnicott et cela fut un moment mutant, décisif, où je me suis remis en question. Je me suis dit qu'il fallait que j'explore d'autres univers théoriques.

Un peu plus tard, après avoir quitté la direction du département de psychiatrie et avoir effectué une recherche sur la relation des enfants asthmatiques à leur mère, j'ai choisi de travailler à plein temps aux soins de jour avec les enfants psychotiques. Et là, ce fut pour moi la redécouverte de Klein : des théories qui me paraissaient absurdes auparavant, m'apparaissaient soudainement très éclairantes pour comprendre le matériel que m'apportaient les enfants psychotiques.

Sur les entrefaites, j'avais été voir à Québec Dr Henri Rey, un grand Kleinien à la retraite, qui venait chaque année enseigner pendant quelques mois. J'ai alors été témoin d'entrevues qu'il faisait avec un jeune psychotique et j'ai été complètement bouleversé par la façon dont il arrivait à communiquer avec lui. Il est venu plus tard à Montréal ; à ce moment-là j' étais directeur de l'enseignement à Sainte-Justine et je me suis organisé pour avoir de fréquents contacts avec lui. Il venait souvent à la maison, et me parlait pendant des heures avec un enthousiasme incroyable de Mélanie Klein, de son enfance à l'île Maurice, de son analyse avec Rosenfeld, de ses rencontres avec Mélanie Klein, de toute son expérience de trente ans au Maudsley Hospital dans le traitement des psychotiques. Il est devenu un très grand ami. Il fut une source d'inspiration et d'admiration pour moi ; il a contribué à fixer mon orientation kleinienne et m'a aidé à comprendre le cas de Sébastien, un enfant psychotique de huit ans que j'avais pris en analyse. J'ai vécu des moments d'enthousiasme... la théorie kleinienne est devenue le courant majeur de ma pensée.

Yvon Gauthier, René Diatkine et Henri Rey sont trois hommes qui ont eu une très grande importance dans l'organisation de ma compréhension du fonctionnement psychique. Il y a d'autres analystes à qui je dois beaucoup aussi : des superviseurs comme Paul Lefebvre, André Lussier, Jean-Louis Langlois et Henry Kravitz m'ont marqué chacun à leur façon. André Lussier est devenu un ami, un modèle. Je dois beaucoup aussi à Paul Lefebvre qui m'avait supervisé temporairement pour des thérapies d'adultes.

L. Q.: Votre mémoire de candidature à l'Institut de psychanalyse de Montréal a porté sur la cure de Sébastien, un des enfants que vous avez rencontré au Centre de jour de Sainte-Justine. N'était-ce pas la première fois qu'une analyse d'enfant faisait l'objet d'un mémoire de candidature à l'Institut psychanalytique de Montréal ? Et la première fois aussi que son orientation théorique était résolument kleinienne?

P. D. : Oui, c'était bien la première fois et cela a été très bien reçu par l'auditoire. Ma candidature a été acceptée à la quasi unanimité des voix. À cette occasion Monique Meloche, dont le dévouement a toujours été grand, a traduit en anglais mon travail pour que $\mathrm{D}^{\mathrm{r}}$ Clifford Scott puisse le lire. J'ai pu avoir ensuite de nombreux contacts avec celui-ci, même si je comprenais à peine, comme beaucoup de mes collègues, la moitié de ce qu'il me disait si rapidement en anglais. C'était, lui aussi, un homme extraordinaire. 
L. Q.: Vous aviez auparavant présenté cette cure au Congrès des psychanalystes de langue romane qui s'est tenu à Montréal en 1982. Mélanie Klein n'avait pas du tout la cote en France à cette époque....

P. D.: Oui, c'est vrai. Et je pense que j'avais été un peu téméraire parce que j'avais présenté la cure de Sébastien, assortie d'un commentaire sur le processus thérapeutique inspiré des travaux de Meltzer. Bien sûr, les Français présents étaient un peu réticents. Diatkine, entre autres, m'avait dit: «Mais on ne fait pas une psychanalyse avec un guide bleu !» et je lui avais répondu : «Écoutez, j’ai lu le guide bleu après avoir fait l'analyse et ça m'a permis de retrouver un grand nombre des paysages que j'avais déjà découverts au cours du voyage.»

L. Q. : Avait-il été satisfait de la réponse?

P. D. : Oui, mais il a été encore plus satisfait lorsque j' ai ajouté des réflexions de Winnicott sur la psychose et le jeu. Finalement les Français ont été beaucoup plus gentils avec moi qu'ils ne le sont entre eux. C'était un congrès où André Lussier était le principal présentateur et moi celui qui avait eu la chance de présenter sur les enfants, alors que le congrès se tenait à Montréal.

L. Q.: À ce moment-là, cette présentation visait à étayer vos réflexions sur le processus thérapeutique et vous avez rapporté un moment mutatif de cette cure, celui où vous avez mis en acte l'impuissance et la colère que ce patient vous faisait vivre. Pouvez-vous nous relater cet épisode et nous faire part de votre compréhension de ce qui s'est passé?

P. D. : Cela faisait des jours et des jours que l'analyse tournait en rond. Sébastien avait une petite calculatrice à laquelle il faisait toujours marquer "erreur», «erreur», «erreur». J'étais las, et un peu découragé; de plus, il enlevait ses chaussures régulièrement pendant la séance et jouait avec elles, sans que je puisse rien en faire, rien interpréter. À un moment donné, m'interrogeant sur mon irritation et mon sentiment d'impuissance, j'ai eu la sensation de comprendre quelque chose, j'ai alors pris une de ses chaussures et je l'ai lancée en haut sur le dessus d'une lampe où il ne pouvait pas l'atteindre. Il m'a demandé sa chaussure mais je ne la lui ai pas donnée. Je lui ai expliqué à ce moment-là qu'il était en train de vivre ce qu'il me faisait vivre à moi. Ne me faisait-il pas toujours marquer «erreur»? Et un peu magiquement, il m'a répondu : «Docteur Drapeau j'ai des bonhommes dans mon ventre.» Cet épisode fut un point tournant; il marqua le début d'un travail extraordinaire à travers le dessin où toute une histoire s'est mise en marche.

Ici il faut bien faire des distinctions, il y avait eu agir de ma part mais un agir très différent de l'acting out; c'était plutôt l'étayage d'une interprétation sur un geste. Ce qui ressemble à ce qui se passe dans le jeu des enfants où, Freud fut le premier à le remarquer, la pensée doit s'étayer sur des objets externes car l'abstraction complète n'est pas possible. Donc, avec des enfants très malades, je dirais aussi avec des adultes très malades, de tels gestes interprétatifs ainsi accompagnés de la parole peuvent peut-être faire bouger certaines choses qui autrement demeureraient inatteignables. 
L. Q. : C'est dire que ce geste de votre part, même s'il était une expression de ce que vous éprouviez, même s'il a dû vous procurer un soulagement, n'était pas essentiellement une façon d'évacuer cet éprouvé?

P. D.: J'avais beaucoup réfléchi à l'impasse dans laquelle nous étions et j'en étais venu à comprendre que Sébastien, probablement par identification projective, me faisait vivre à moi une impuissance qu'il avait vécue lorsqu'il était tout petit et qu'était ainsi remis en scène dans le transfert quelque chose d'un lointain passé; je lui traduisais un peu ce mécanisme dans l'interprétation que je lui apportais. C'est sûr et certain que de mon côté, il y avait de l'impatience et même de la colère, mais $\mathrm{j}$ 'avais bien examiné si ces sentiments étaient tout simplement dus à une angoisse de castration provoquée par mon impuissance comme thérapeute ou s'ils pouvaient exprimer un phénomène plus profond. Je pense qu'il fut essentiel, pour sortir de l'impasse, de comprendre la dimension de l'identification projective impliquée dans l'échange avec ce jeune patient, tout en reconnaissant aussi ce qui venait aussi de moi.

L. Q.: En somme, on pourrait dire que vous avez décidé de lui laisser voir quelque chose de ce qu'il vous faisait vivre et que vous compreniez par ailleurs?

P. D.: Oui. C'est ce qui s'est passé. Mais, honnêtement, quand je l'ai fait, je ne me doutais pas de l'effet immédiat que pouvait avoir cette interprétation agie. J'avais vécu tellement d'échecs jusque-là. Sa réponse fit renaître en moi l'espoir - qui est aussi nécessaire au thérapeute - et m'a beaucoup appris. Lors de la présentation à l'auditoire français, j'ai raconté cet événement avec hésitation, mais quand je suis allé en France, l'année suivante, on m'appelait «l'homme à la chaussure $\gg . .$.

L. Q.: Cet épisode a marqué l'imaginaire des auditeurs tout autant que la cure elle-même...

P. D. : Tout à fait, mais elle m'a aussi permis d'établir des relations enrichissantes avec de grands analystes français: Lebovici, Soulé, Kreisler, Chiland et plus tard Anzieu, et surtout Florence Bégoin-Guignard

L. Q. : Lorsque, deux ans plus tard, vous avez repris le récit de cette cure qui s'était poursuivie entre-temps pour en faire l'objet de votre mémoire de candidature, vous avez mis l'emphase sur la description et la théorisation du passage de la représentation quasi directe de fantasmes archaïques à leur expression de plus en plus métaphorisée, du passage du corps à corps au désir, de l'équation symbolique au symbole.

Dans ce texte intitulé: «Les contes de la folie. Réflexions sur le processus thérapeutique », vous écrivez que c'est chez Hanna Segal et Winnicott que vous avez trouvé les explications les plus éclairantes de ces phénomènes. Pouvez-vous nous en parler?

P. D.: Dans un article sur la formation du symbole, Hanna Segal montre bien comment, au cours de la position dépressive, le symbole est une création, une représentation créée qui vient remplacer la représentation de l'équation symbolique, laquelle est une copie concrète, peu différenciée de l'objet lui-même. 
Winnicott décrit de façon plus imagée le même phénomène lorsque, se référant aux processus transitionnels, il montre comment l'enfant a progressivement accès à la symbolisation dans le jeu. Après avoir mieux compris ce phénomène, mon désir d'interpréter et de guérir a été en partie remplacé par celui, plus modeste, d'arriver avec Sébastien à la capacité de jouer. Pour atteindre cet objectif, je me suis largement servi du dessin exécuté en parallèle avec lui. La thérapie s'est donc recentrée sur l'accès au jeu et à la symbolisation. Dans une telle démarche, nous sommes impliqués plus activement comme thérapeutes, car il nous faut apporter nous-mêmes un matériel qui sert de catalyseur à la symbolisation en étayant notre intervention sur la concrétude du jeu ou du dessin. J'ai l'impression que cette prise de conscience a influencé ma façon d'être dans toutes les thérapies que j'ai conduites par la suite.

L. Q. : La créativité résulterait-elle du passage de la position schizo-paranoïde à la position dépressive ou serait-elle l'instrument qui favoriserait ce passage?

P. D.: Je crois qu'elle en est l'instrument. Le passage à la position dépressive est le passage d'une position où l'angoisse est centrée sur la peur d'être attaqué et détruit à une position où l'angoisse devient celle de protéger l'objet qu'on aime, lequel risque d'être détruit par notre propre agressivité. La création de symboles qui représentent l'objet tout en étant différents de lui permet de protéger l'objet et rend possible le passage à la position dépressive.

L. Q. : La création de symboles permettant en quelque sorte de protéger l'objet aimé que l'on veut conserver grâce au déplacement sur eux de l'agressivité éprouvée envers l'objet?

P. D.: Oui. D'une certaine façon le symbole peut être remplacé par un autre, il permet le déplacement. L'utilisation du symbole permet aussi l'accès à une façon nouvelle de réparer l'objet, de passer de la réparation concrète, itérative et sans fin observée dans les positons maniaque et obsessionnelle à la réparation symbolique, qui se distingue de la précédente par sa créativité et l'apaisement qu'elle procure. L. Q.: La cure de Sébastien constitua aussi pour vous le point de départ d'une réflexion approfondie sur les fantasmes originaires, parallèlement à une autre sur les théories sexuelles infantiles. Au terme de votre long périple avec les enfants psychotiques qui interrogent au plus près nos conceptions de l'origine de la vie psychique et de l'inconscient, avez-vous le sentiment d'y voir un peu plus clair? P. D. : Je ne suis pas certain d'y voir vraiment clair... C'est là une question fondamentale qui dépasse de beaucoup mes connaissances; je peux seulement parler des questions que je me pose sur ce sujet qui m'a toujours vivement interrogé. Il y a déjà plusieurs années, j'ai écrit un texte intitulé «Des théories et des dragons » où je parle, évidemment, des théories sexuelles infantiles de Freud et des dragons de Mélanie Klein. J'y formulais l'hypothèse que les théories sexuelles infantiles ne découlaient pas nécessairement d'une structure psychique transmise par phylogenèse mais pouvaient résulter de la métaphorisation des fantasmes primitifs découverts par Klein chez les enfants beaucoup plus jeunes. Il y aurait continuité génétique entre le concept de mort de la mère dans la position dépressive et le 
concept de meurtre du père dans la conflictualité œedipienne. Mon expérience en psychiatrie et en psychanalyse des enfants m'a bien montré que la structuration œdipienne est l'aboutissement d'une longue conquête; j'ai vu des centaines d'enfants où cette conquête n'a jamais eu lieu ou a cessé bien avant d'être arrivée au but. J'ai aussi vu beaucoup d'adultes chez qui ce processus s'est figé en cours de route et où s'est installé un pseudo Edipe.

Par ailleurs, chez ceux qui ont atteint la position œdipienne, nous arrivons à un noyau, à un conflit structurant dans l'inconscient qui se répète toujours, pour l'essentiel, de la même façon, même si les circonstances du développement sont souvent profondément différentes. Le père du petit Hans était un homme très bon et très doux et, pourtant, dans l'inconscient de Hans, il jouait le rôle du père tyrannique de la horde primitive qui appelle au meurtre. Quelque chose, ici, échappe à l'observable et à la logique du développement, quelque chose semble se répéter qui dépasse les simples conséquences de l'événementiel, comme si une structure innée, comme nous le dit Freud, était plus forte que la réalité vécue. D'une position développementaliste nous passons à une position structuraliste. Je dois dire que mon expérience dans plusieurs analyses d'adultes me semble aller dans ce sens. Malgré ma réticence à admettre une telle hypothèse, je crois que peut-être l'interaction fantasmatique mère-enfant, de génération en génération, pourrait mieux expliquer la présence de ces fantasmes organisateurs communs.

L. Q.: Vous dites que votre expérience avec les adultes vous a amené à opter de plus en plus fermement pour le point de vue structuraliste, les facteurs externes ne vous paraissant pas suffisants pour rendre compte de l'intensité du conflit observé. Pouvez-vous expliciter cette prise de position?

P. D. : Autant mon expérience avec les enfants m'a convaincu du rôle déterminant du vécu, de l'expérienciel, de la continuité génétique, autant mon expérience en analyse avec des adultes de structure névrotique m'a amené à croire à l'importance du point de vue structuraliste. La grande majorité de mes analysants adultes avait atteint pour une bonne part la position dépressive et la conflictualité œdipienne, et chez eux j'étais à même d'observer le conflit œdipien plus directement que je n'avais pu le faire en travaillant avec les enfants. Ainsi j'en suis venu progressivement à penser qu'un autre facteur était peut-être nécessaire pour expliquer l'aboutissement de la continuité génétique dans une structure œdipienne unique, si identique d'un cas à l'autre malgré les différences importantes dans l'environnement. Il faut se souvenir que le plus refoulé n'est pas nécessairement le plus archaïque. On a facilement tendance à oublier le rôle central que joue l'Edipe dans la détermination de la dynamique inconsciente, surtout lorsque certains fantasmes archaïques viennent brouiller le tableau et accaparent notre attention.

L. Q.: Vous avez écrit quelque part que les théories tentent d'apporter des réponses aux énigmes, mais que la théorie doit ouvrir sur de nouvelles énigmes. Cette idée du danger d'enfermement dans un seul système de compréhension semble prégnante chez vous. Dans la conférence publique de la Société psychanalytique de Montréal que vous avez donnée et qui s'intitulait «Hans, mon frère 
Hans, ne vois-tu rien venir?», vous avez tenté de présenter l'aventure de l'enfant dans la psychanalyse comme une grande fresque où se juxtaposaient les tableaux qu'en dessinent Freud, Klein et Winnicott. Il est impossible de rendre compte de cette fresque dans le cadre de cet entretien, aussi vous demanderais-je, s'il est possible, en vous situant au-delà des éléments précis de leur doctrine, de cerner des points organisateurs de la pensée de chacun d'eux?

P. D.: Avant de répondre, je pense qu'il est important de préciser mes appartenances théoriques. Je suis avant tout un fils de Freud, parce que c'est là que m'ont conduit mon analyse et ma formation, mais la rencontre des enfants psychotiques m'a ensuite entraîné à me centrer sur Klein, les post-Kleiniens et, finalement, à partir de mon expérience clinique, sur Winnicott. Dans ma rencontre avec ces auteurs, ces nouveaux maîtres, j'ai toujours cherché à établir les liens qui pouvaient relier leurs théories à celle de Freud. J'ai beaucoup écrit sur la relation Winnicott-Freud à partir de la description des enfants mythiques qu'ils ont respectivement élaborés et j'ai essayé de démontrer à quel point les deux théories étaient complémentaires.

Pour répondre directement mais schématiquement à votre question, je crois que pour Freud le complexe d'Edipe et l'angoisse de castration demeurent tout au long de son œuvre les grands organisateurs. Il faut retenir aussi la conception freudienne du travail analytique comme partagé entre deux pôles, celui de l'analyse et de la déliaison d'une part, et celui de la construction, d'autre part. C'est l'histoire de ma propre analyse.

Klein, grâce à l'analyse des enfants, fait un bond prodigieux. Pour elle, l'essentiel se joue dans le passage de la position schizo-paranoïde à la position dépressive, dans le passage d'une angoisse de persécution par l'autre à une angoisse de perdre l'autre, de détruire un autre qu'on aime par sa propre agressivité. De là l'importance de la capacité de réparation symbolique et de la création. En permettant la régression et en interprétant la haine dans le transfert, l'analyste faciliterait la réparation symbolique et l'élaboration de la névrose infantile

Winnicott a introduit l'indispensable relation à l'autre. Sa conception de l'analyse est essentiellement basée sur sa compréhension du développement de l'enfant en relation avec la mère et les processus transitionnels. Mais il est tout aussi important de voir qu'il a aussi ajouté une dimension nouvelle dans la compréhension du développement. Il adhère à la théorie freudienne pour tout ce qui concerne le développement pulsionnel, le «Faire» qui relève du masculin dans sa propre métapsychologie, mais il est davantage intéressé par la dimension de l' «Être », du Self et de la création qui relèverait selon lui du féminin.

En se référant au début du sentiment d'être, il décrit l'angoisse liée à la crainte de l'effondrement. Par contre, lorsqu'il examine un niveau plus avancé de la relation à l'autre, il insiste sur l'angoisse d'intrusion et de perte du vrai self. Dans la relation avec le thérapeute, une des plus grandes angoisses du patient est, selon lui, l'angoisse d'intrusion soulevée par les tentatives du thérapeute de le comprendre et par ses interprétations prématurées. De là, en plus de la nécessité de la 
relation de sécurité donnée par la stabilité du cadre et le holding par l'interprétation, la nécessité de la non intrusion et du soutien de la créativité du patient. C'est là que m'a conduit mon expérience en thérapie.

Dans certaines analyses, de façon paradoxale, la crainte d'intrusion peut être à l'origine de défenses excessivement tenaces de repli là où il pourrait y avoir régression. Winnicott nous rappelle que l'amour peut, aussi bien que la haine, être cause d'intrusion.

L. Q.: Pourrait-on dire que pour Winnicott, l'angoisse d'intrusion joue un rôle organisateur analogue à celui que Mélanie Klein attribue à l'angoisse dépressive et Freud à l'angoisse de castration?

P. D.: Pas de la même façon. Dans le développement de l'enfant l'angoisse d'intrusion doit être surmontée pour donner accès à la capacité d'être seul en présence de l'autre, pour ouvrir sur la capacité de communiquer, d'échanger, de jouer avec lui sans avoir peur de devenir sa possession et de perdre son identité. Dans la thérapie, nous assistons au même scénario. Probablement que Winnicott a beaucoup souffert pendant sa deuxième analyse du bombardement interprétatif que lui faisait subir son analyste kleinienne Joan Riviere. Ainsi en est-il arrivé à parler de «sanctuaires qui ne doivent pas être violés».

L. Q.: Vous savez que Piera Aulagnier pose le «droit au secret» comme une condition pour pouvoir penser et insiste sur la nécessité de respecter ce droit afin de préserver l'autonomie psychique. N'est-il pas intéressant de relever ce point de convergence chez des auteurs aux allégeances théoriques bien différentes?

P. D.: Tout à fait.

L. Q.: Lors de congrès internationaux, vous avez été appelé à commenter les présentations cliniques d'autres analystes. Considérant la névrose infantile comme une tentative d'élaboration des angoisses primitives, comme le veut la théorie kleinienne, vous avez proposé dans ces commentaires une lecture qui tenait davantage compte des accrochages dans la relation primitive à la mère. Dans les cures de patients non psychotiques, quelle importance accordez-vous à l'interprétation des noyaux les plus profonds de l'organisation psychique?

P. D. : Si l'on admet que la névrose infantile est une élaboration, une perlaboration des positions psychotiques infantiles, il faut toujours, dans l'approche de la névrose, rester très conscient des accrochages possibles dans le parcours de la position dépressive. L'atteinte de la position dépressive est un travail qui dure pratiquement toute la vie et qui n'est jamais terminé. Donc chez ceux qui ont des conflits névrotiques, nous rencontrerons régulièrement des accrochages dans l'élaboration de la position dépressive. Vu sous cet angle, le contenu fantasmatique peut révéler un sens autrement invisible.

Dans mon commentaire sur la présentation de l'américain Steven Luria Ablon, lors d'un congrès de l'Association internationale de psychanalyse, j'avais souligné le besoin qu'avait l'enfant de réparer son thérapeute tout au long de la thérapie. Cette dimension avait complètement échappé à l'analyste qui, par ailleurs, avait fait un excellent travail. Hannah Segal était intervenue dans le même sens que moi, 
ce qui m'avait, bien sûr, apporté un grand réconfort... Je pense qu'il faut toujours être sensible à cet aspect avec les adultes névrotiques. Cette conception de la névrose comme provenant d'un accrochage dans l'élaboration d'une position psychotique tempère aussi la peur que l'on peut avoir de certains fantasmes primitifs, lesquels ne signifient pas nécessairement la présence d'une pathologie très grave. L. Q. : Préconisez-vous l'interprétation systématique des noyaux les plus profonds?

P. D.: Non, absolument pas, à moins que le matériel nous y conduise ou que ces noyaux puissent expliquer une partie des comportements ou des symptômes. Souvent nous constatons qu'il y a eu chez certaines personnes, de façon étrange, comme un clivage, une mise à part, un encryptement de noyaux primitifs les empêchant d'avoir des effets dévastateurs immédiats sur l'évolution psychique. C'est parfois plus tard lors d'un traumatisme important, que ces dragons endormis se réveillent. La construction des structures inconscientes ne se fait pas de façon mécanique selon une logique qui correspondrait toujours à nos hypothèses théoriques. À ce sujet, Bergeret a produit des textes très intéressants où il montre comment certains noyaux pathologiques avec lesquels la personne a pu s'accommoder en grande partie n'entraînent pas nécessairement un arrêt visible du développement psychique. Plusieurs mécanismes d'accommodation sont possibles et dans leurs études sur la continuité génétique, les néo-kleiniens ont insisté de façon paradoxale sur ces mécanismes.

À titre d'exemple, j'ai connu des personnes qui sont devenues d'excellents thérapeutes en dépit de fixations à des noyaux primitifs dont ils avaient réussi à bien se protéger.

L. Q.: Vous avez passé des années à approfondir les pensées de Klein et de Winnicott. Qu'est-ce qui vous a fait privilégier ces auteurs?

P. D. : J'ai en effet consacré de longues heures à lire, méditer et analyser la pensée de ces auteurs, de même que les développements qu'y ont apportés les Meltzer, Bion, Segal, Rosenfeld, Green, Roussillon, Jeammet, Begoin, Guignard pour n'en citer que quelques-uns.. Je pense que le fait de travailler avec des adultes et des enfants a joué un rôle important dans le choix des auteurs que j'ai privilégiés. Je suis venu à Klein et Winnicott à travers l'approche des enfants dans mon travail à Sainte-Justine. Dans un second temps, ces auteurs, surtout Winnicott, m'ont beaucoup influencé dans ma pratique analytique auprès des adultes.

Mais votre question pourrait nous amener beaucoup plus loin. Pourquoi le choix de ces auteurs en particulier, j'aurais pu continuer à privilégier des auteurs de la psychologie du Moi ou m'orienter du côté de Lacan, par exemple... Et, si on pousse plus loin la question, pourquoi cette fascination pour la psychose? pourquoi le choix des enfants? pourquoi les deux types de pratique? Je pourrais raconter des anecdotes : les enfants psychotiques que j'avais vus en accompagnant mon père dans ses visites aux malades... certains tableaux... certaines histoires entendues.. certains poèmes... Ce n'est pas là que se trouve la vraie réponse mais plutôt dans l'idéalisation, la défense contre la théorie du maître, le besoin de 
réparer, etc. Toutefois n'est-il pas intéressant de constater que les plus grands maîtres, dans leurs découvertes, se sont pour ainsi dire servis des pathologies qui les habitaient — qu'on songe à la névrose de Freud, à la dépression de Mélanie Klein, à l'identification féminine de Winnicott — comme points de départ, comme catalyseurs de leur réflexion? Que s'est-il passé pour moi, de quel poids ont pesé mes conflits personnels, je serais bien présomptueux de vouloir répondre à cette question. L'inconscient a sûrement agi, mais la grande valeur de ces théories, leur grande utilité pour comprendre et soigner ont certainement joué un rôle déterminant. En fin de parcours, je puis dire que Winnicott est l'auteur auquel je me suis le plus naturellement identifié.

L. Q. : Dans De Freud à Winnicott, les enfants mythiques, vous soutenez la thèse que Winnicott a développé, en l'enrichissant, le versant moïque de la première théorie freudienne des pulsions. Pouvez-vous nous exposer succinctement cette thèse?

P. D.: C'est une hypothèse à laquelle je suis arrivé après avoir tenté de comparer les modèles d'enfants mythiques qui se retrouvent dans les œuvres de Freud et de Winnicott. Cette démarche m'a conduit à désigner la première théorie des pulsions - qui oppose les pulsions sexuelles aux pulsions d'autoconservation - comme «fondement» possible, point de départ de la théorie winnicottienne. Je souligne que je parle de la théorie des pulsions et non des première et deuxième topiques freudiennes, car Winnicott se sert continuellement des concepts de la deuxième topique tout en continuant de se référer à la première théorie des pulsions. À titre d'exemple, il divise autrement l'espace psychique formé par la triade Ça, Moi et Surmoi en ajoutant le Self à ces trois instances, ce qui est tout à fait fondamental pour rendre compte de ses découvertes.

Mais je voudrais d'abord revenir brièvement sur quelques éléments qui soutenaient mon propos. L'étude des enfants mythiques construits par Freud : enfant du rêve et de la névrose, enfant de la psychose et du narcissisme, enfant de la préhistoire, enfant de la métapsychologie, enfant du trauma et de l'au-delà du principe du plaisir, nous montre que Winnicott a rencontré les mêmes problématiques que Freud mais, qu'à partir de son expérience avec les enfants et les psychotiques, il y a apporté d'autres réponses en se référant à certaines dimensions du Moi ou en prolongeant la notion de pulsion du Moi,

Le premier argument reposait sur le fait que Winnicott n'a jamais accepté la pulsion de mort, qui implique une force fondamentalement négative au sein de l'être humain. Pour Winnicott, il n'y a pas, au départ, de force fondamentalement négative: l'agressivité fait partie de l'amour; comme le feu, elle peut avoir des effets positifs ou négatifs selon la réponse de l'environnement. Pour lui comme pour Freud, dans la première théorie des pulsions, l'agressivité est une force de vie qui donne l'énergie à la pulsion libidinale. C'est d'ailleurs exactement ce que soutenait Freud, lors d'un commentaire théorique qu'il faisait dans son texte sur le Petit Hans, pour réfuter l'hypothèse adlérienne d'une pulsion d'agression et défendre le droit à chaque pulsion d'avoir en elle, comme il l'écrit, ce qui lui donne sa force, ainsi que son statut de pulsion. 
Évidemment, Winnicott donne au concept d'autoconservation une dimension plus large que la survie : l'essentiel à ses yeux est la créativité du Moi qui donne toute sa richesse au développement humain. C'est dans ce sens que je parle de point de départ et de dépassement.

Dans sa première théorie, Freud parlait de la relation de tendresse qui existe entre une mère et son enfant et qui sert d'étayage à la pulsion sexuelle. Winnicott va dans le même sens lorsqu'il jumelle les relations à la mère pulsionnelle et à la mère-environnement.

Dans le cadre de la première théorie des pulsions, Freud a développé la notion fondamentale de narcissisme, ajoutant une dimension nouvelle au Moi qui peut investir la libido sur lui-même ou dans les objets. Plus tard, dans ses études métapsychologiques, il parle pour la première fois de «Moi-sujet». Sur ce point aussi, Winnicott a prolongé la réflexion de Freud en se recentrant sur une autre dimension du Moi-sujet, en parlant de Self et de la qualité essentielle du sentiment «d'être, d'être vivant, d'être réel et d'être vrai».

Ajoutons enfin que Freud, dans Totem et Tabou, réfère à l'illusion de toutepuissance magique chez l'homme primitif de la période animiste en l'associant à un surinvestissement libidinal du Moi, alors que Winnicott, lui, parlera de la nécessaire illusion de toute-puissance du Moi infantile attribuable à la fausse perception qu'il a de sa puissance, grâce au holding de la mère.

En introduisant la deuxième théorie des pulsions, Freud, dans Au-delà du principe de plaisir, après avoir parlé de tentative de maîtrise dans le jeu de la bobine, en arrivera à une force de répétition liée à la pulsion de mort ou d'auto-destruction. Winnicott interprétera ce jeu comme une tentative de réparation, donc comme une marche vers la vie. Beaucoup plus tard pour, selon ses dires, libérer Freud du poids de la pulsion de mort, il donnera une autre explication de la destructivité dirigée contre soi-même, Cette auto-destructivité serait la conséquence d'une mauvaise réponse de l'environnement à l'agressivité de l'enfant qui est ainsi détournée de son but premier et pour ainsi dire «pervertie» en une force contraire; elle pourrait être aussi le résultat d'un auto-empoisonnement dû aux restes toxiques de traumatismes non mentalisés qui n'ont pas eu accès à la représentation dans l'espace psychique.

C'est ainsi que j'ai tenté de montrer que Winnicott s'était posé les mêmes questions que Freud, mais que son expérience de l'enfant et de la psychose lui avait permis de prolonger la réflexion de ce dernier en se servant du versant moïque de la première théorie des pulsions, c'est-à-dire sans recourir à la notion de pulsion de mort. Autant Freud a élaboré sur le destin des pulsions sexuelles, autant Winnicott a centré ses recherches sur un Moi animé par une force créatrice permettant l'accès à la symbolisation à travers le jeu. Autant Freud avait insisté sur le refoulement pulsionnel, autant Winnicott insistera sur la dissociation du moi. En parallèle à l'inconscient du refoulé, il proposera un inconscient du manque, du non-représenté ou non-symbolisé dans l'espace psychique.

En terminant sur ce point très important pour moi, je tiens à souligner que j'ai voulu démontrer dans ce long travail que la théorie winnicottienne était 
complémentaire à celle de Freud. En parodiant Winnicott, j'ai écrit que «Winnicott sans Freud ça n'existait pas». Si cette étude comparative des deux théories était si nécessaire pour moi, c'est parce que toutes les deux inspirent profondément ma pratique.

Dans les jours qui ont suivi mon accident cérébrovasculaire, mon ami Jacques Mauger m'a apporté un livre de Laurence Kahn, une grande spécialiste de Freud, intitulé Cures d'enfance. À partir de sa clinique, elle fait une synthèse merveilleuse de l'approche de Freud et de Winnicott. Ce cadeau ne pouvait venir à un meilleur moment.

L. Q.: Attardons-nous quelques instants, si vous le voulez bien, aux pulsions d'autoconservation. Dans la vision freudienne, ces pulsions correspondent à des montages innés qui assurent la survie de la vie animale en nous. Mais dans la vision winnicottienne, disiez-vous, l'autoconservation ce n'est pas seulement survivre et s'adapter, c'est vivre...

P. D.: Et créer. La créativité est, finalement, la chose la plus fondamentale chez Winnicott. Dans un des seuls textes qu'on pourrait qualifier de métapsychologiques que Winnicott ait écrit, il en arrive à distinguer le féminin du masculin mais d'une façon bien particulière. Au masculin est attaché tout ce qui est pulsionnel, tout ce qui est «faire» et au féminin, tout ce qui relève de l'être et de la créativité. Il ne parle pas de sublimation mais d'une pulsion créatrice fondamentale au niveau du moi ; il ira même jusqu'à parler d'orgasme du moi. C'est dans ce sens que j'ai l'impression qu'il a poussé plus loin la notion d'autoconservation en la transformant en une force qui ressemble à ce que Freud avait déjà décrit comme pulsion de vie.

Winnicott s'est toujours dit fidèle à Freud et je pense qu'il s'est inspiré de l'ensemble de son œuvre. Dans sa correspondance cependant, il écrivait qu'il connaissait mal Freud et qu'il ne pouvait supporter les discussions métapsychologiques, qu'à l'Institut Britannique, il avait beaucoup de peine à suivre lorsque les discussions étaient trop centrées sur le conflit œdipien. Mais Winnicott est un génie qui a ouvert un champ inouï avec sa centration sur le Self, l'être, la relation mère-enfant et le concept de transitionnalité. Dans nos analyses, l'accès à la créativité est une dimension essentielle à considérer, par-delà les conflits pulsionnels.

L. Q. : J'ai bien aimé la comparaison que vous faisiez, dans un de vos articles, entre Freud et Winnicott. Vous y écriviez que Freud pouvait être considéré comme un scientifique qui essayait de comprendre comment fonctionnaient les choses et Winnicott, lui, pouvait être comparé plutôt à un jardinier, à quelqu'un qui se demandait ce qu'il fallait faire pour conserver la vie des êtres.

P. D.: Cette idée de comparer Winnicott à un jardinier s'est imposée à moi au terme de ma fréquentation de son œuvre. Il m'est un jour revenu à la mémoire un passage de Terre des hommes de Saint-Exupéry où cet auteur parle de la rencontre qu'il a faite dans un train avec un couple d'ouvriers très pauvres qui tenaient dans leurs bras un très bel enfant. Dans un autre environnement, cet enfant serait peut- 
être devenu un être d'exception mais parce qu'il n'y a pas de jardinier pour les hommes comme il y en a pour les roses, son avenir serait vraisemblablement beaucoup plus terne. Il serait, selon sa magnifique expression, un Mozart assassiné. J'ai pensé que le point de vue de Winnicott rejoignait les préoccupations de Saint-Exupéry.

Winnicott thérapeute de génie est bien du côté du jardinier à condition de ne pas le faire passer pour une nounou car il était guidé non pas simplement par des sentiments de tendresse mais par une théorie précise. Par le holding interprétatif, Winnicott espérait libérer les forces créatrices susceptibles de permettre la reprise d'une marche naturelle vers la guérison. Meltzer s'est sûrement beaucoup inspiré de lui dans sa description du processus analytique, qu'il considère comme un processus naturel qui se développe si on instaure et maintient le cadre analytique.

Winnicott, comme Freud dans la première théorie des pulsions, était profondément influencé par les idées de Darwin sur l'évolution. Tous les deux croyaient en une progression vers l'avant naturelle et nécessaire, seuls les accidents dans l'environnement pouvant faire dévier, freiner l'évolution. Ces accidents étaient pour Freud le manque, le trop, la séduction et pour Winnicott, les déficiences dans le holding, dans la présentation de l'objet et l'intrusion. Cette force «créatrice» qui sous-tend l'évolution des espèces, Winnicott la voyait aussi à l'œuvre dans le développement de l'enfant et la progression du processus thérapeutique.

La deuxième théorie des pulsions introduit dans le devenir de l'être humain une lutte entre deux forces qui s'opposent: pulsions de vie, pulsions de mort ou d'autodestruction. Dès lors, deux principes philosophiques différents pourront guider les thérapeutes. Le premier, pessimiste, où il y a le jeu de la pulsion de mort: chez Klein, ça va jusqu'au point où la pulsion de mort est trop grande, le progrès devient presque impossible et il y a chute dans la psychose. Winnicott se situe nettement du côté d'une deuxième philosophie, optimiste, où le progrès est toujours possible, la pathologie étant attribuée à des arrêts de développement, à des accrochages dus aux manques de l'environnement et non pas à l'action d'une force fondamentalement auto-destructrice. Je dois admettre qu'il est plus facile pour moi d'adhérer à la deuxième perspective, même si, face à certains échecs thérapeutiques, on peut toujours se demander si le pessimisme de Freud n'avait pas aussi des fondements tout à fait réels.

L. Q.: Que pensez-vous des travaux actuels sur l'attachement? Considérez-vous l'attachement comme la manifestation d'une pulsion d'autoconservation ou d'une pulsion sexuelle?

P. D.: J'attache une très grande importance aux travaux actuels sur l'attachement. Je crois que Bowlby avait raison de s'inspirer de l'éthologie et de l'appliquer à l'homme; j'imagine que si Freud avait connu l'éthologie, il l'aurait aussi utilisée et je suis convaincu que l'attachement aurait fait partie des pulsions d'autoconservation. Les cliniciens de l'attachement ont développé une technique d'observation beaucoup plus rigoureuse et objective du développement de l'enfant et qui peut certainement nous apporter beaucoup. 
Depuis plus de deux ans déjà, je dirige des séminaires sur les relations entre la théorie analytique et celle de l'attachement. À l'instar de Peter Fonaggy, auteur d'un livre sur ce sujet, j'estime que les observations et les déductions des théoriciens de l'attachement ne font que confirmer nos théories psychanalytiques. Elles vont, en effet, dans le même sens que les théories de la relation d'objet, les théories néo-kleiniennes et, aussi, que ce qu'on appelle aujourd'hui la troisième topique. Elles recoupent la notion winnicottienne de «mère environnement», mais il ne faut pas oublier que Winnicott parlait aussi de «mère pulsionnelle», ce qui à mes yeux est absolument essentiel et permet une approche beaucoup plus complète de la psychopathologie.

Je trouve, par ailleurs, que le terme «attachement» est devenu un passe-partout trop facilement employé. Il permet peut-être à des personnes qui n'ont pas de formation en approche psychodynamique de faire des interventions thérapeutiques, mais il conduit à des simplifications très regrettables car, en s'y référant exclusivement, on en vient à ignorer l'existence du conflit œedipien et le rôle central de l'agressivité.

Je crois aussi que ces travaux décrivent dans un autre langage ce qui a déjà été décrit en psychanalyse. À titre d'exemple: les modèles internes opérants chez les jeunes enfants qui correspondent en grande partie à ce que la psychanalyse a décrit comme des premiers fantasmes organisateurs. Malheureusement, les théoriciens de l'attachement, pourtant souvent des analystes, n'établissent pas beaucoup d'équivalences entre les deux et pour cette raison la lecture de leurs travaux m'a laissé bien souvent sur mon appétit.

L. Q.: Ce postulat des modèles internes opérants confirme les hypothèses analytiques mais vous estimez que d'autres observations et déductions des théoriciens de l'attachement vont dans le même sens?

P. D.: Oui. Il y a aussi le dégagement de modèles d'interaction mère-enfant qui confirment l'importance qu'accordait à la relation mère-enfant les théories d'Anna Freud, de la psychologie du Moi, de Winnicott, d'Aulagnier et de la grande majorité des analystes d'aujourd'hui. Toutefois, lorsque les théoriciens de l'attachement essaient d'expliquer ces modèles, ils n'arrivent pas à distinguer mieux que les analystes ce qui relève du biologique ou de la génétique et ce qui est dû à l'environnement. Bien sûr ils accordent un poids prépondérant à l'environnement, mais quand ils poussent les discussions plus à fond, ils hésitent, tout comme les analystes, entre ce qui est dû à l'environnement et ce qui est dû à la structure de base.

Mais il ne me semble pas intéressant de nous attarder à chercher les éléments qui pourraient nous empêcher de faire profiter l'une de l'autre ces théories qui ont des méthodologies et des modes d'approche très différents. L'important pour moi, c'est l'absence de contradiction fondamentale entre les deux modèles. Les théoriciens de l'attachement nous font découvrir de nouvelles façons de regarder, de penser le développement et la psychopathologie. En ce sens elles ne peuvent que nous enrichir, mais nous avons beaucoup de chemin à faire de part et d'autre Je 
n'ai pas tout lu et, pour le moment, je demeure sur mon appétit quant aux relations qu'elles établissent entre le développement précoce et la psychopathologie adulte. Je ne comprends pas non plus pourquoi elles n'attachent pas plus d'importance au contre-transfert du thérapeute, à la différence des sexes et à l'agressivité.

En revanche, je déplore le sort que les analystes ont réservé à Bowlby, ainsi que leur manque d'intérêt pour les théories de l'attachement. Ils qualifient ces dernières de réductionnistes mais ne pourrait-on pas, à l'inverse, reprocher à la psychanalyse la trop grande complexité de certaines de ses élaborations devenues inaccessibles à la majorité des thérapeutes?

L. Q. : Vous parliez à l'instant des relations qu'il y a lieu d'établir entre les aléas du développement précoce et la psychopathologie adulte; celles que la théorie psychanalytique a supposé être à l'origine de la psychose conservent-elles à vos yeux toute leur pertinence, compte tenu des récentes découvertes de la biologie?

P. D.: Je voudrais d'abord faire une distinction qui me paraît essentielle entre la psychose, l'autisme et les conséquences d'une carence extrême. La classification du DSM IV ne parle plus de psychose, de dysharmonies du développement ou de prépsychose et ceci peut entraîner beaucoup de confusion. La catégorie du trouble envahissant du développement non spécifié est devenue le fourre-tout où se chevauchent plusieurs pathologies différentes.

Sébastien, l'enfant que j'ai suivi en psychanalyse et sur le traitement duquel j'ai écrit, était un enfant intelligent et psychotique. Avec lui j’ai vécu une expérience unique qui m'a permis de saisir directement des mécanismes psychotiques qu'on retrouve chez les schizophrènes adultes.

En ce qui concerne l'étiologie de la psychose, je pense qu'il faut sortir du fameux débat opposant organicité et psychogénèse. Je crois que des facteurs organiques interviennent dans des pathologies aussi graves qui débutent aussi tôt dans la vie. Ces facteurs qui perturbent le développement de l'enfant peuvent entraîner chez les parents vulnérables des comportements qui sont aussi à l'origine de la pathologie. Il y a donc souvent la rencontre de deux ordres de facteurs, mais je peux dire que dans mon expérience j'ai plutôt rencontré des parents remarquables qui, selon moi, ont sauvé leur enfant d'une évolution vers une structure très pathologique. Quelques-uns diront que, malgré leur bonté, certains parents ont inconsciemment entraîné leur enfant dans la psychose; le doute est toujours possible, mais les inquisiteurs, à partir de leurs croyances, accusaient de sorcellerie certaines femmes qui, sans qu'elles en soient conscientes, étaient possédées par le démon...

La littérature nous présente des histoires où le comportement particulier des parents semble bien avoir eu un rôle décisif dans la genèse de la psychose: narcissisme extrême de la mère, exclusion du père, etc. Je crois que dans certain cas on aurait tort de nier le rôle déterminant des facteurs psychologiques mais je crois aussi qu'il faut être très prudent avant de trop généraliser. Par le passé, je pense, certains thérapeutes ont injustement fait porter aux parents une responsabilité «théorique» qu'ils ne méritaient pas. 
C'est une question qui m'a toujours beaucoup intéressé. Il y a déjà plusieurs années j'ai organisé avec Henri Rey un séminaire au cours duquel nous avons étudié le matériel qu'apportait un enfant psychotique et le matériel recueilli de façon concomitante dans la cure analytique de la mère. Ce fut une aventure excessivement intéressante, d'autant plus que les participants étaient des analystes aussi expérimentés et perspicaces que André Lussier et Jean-Louis Langlois. L'interaction fantasmatique était évidente comme elle l'aurait été dans toute relation mère enfant mais je ne crois pas que nous ayons pu tirer des conclusions sur le plan étiologique.

Je demeure quand même aussi impressionné par le fait qu'en général, selon mon expérience, des enfants ayant vécu dans des conditions excessivement difficiles n'ont pas développé de psychose, même s'ils sont restés très marqués dans certains domaines.

Je me rends compte que ma réponse exprime mes hésitations et mes incertitudes. Je pense que les opinions sur l'étiologie de la psychose dépendent largement du point d'observation où nous nous situons. Si un analyste travaille avec un nombre restreint de psychotiques et qu'il peut, dans certaines circonstances, mener une étude très approfondie sur eux, il est fort probable qu'il pourra découvrir des éléments, habituellement non accessibles à une simple évaluation, qui mettront en relief des facteurs psychologiques possiblement en cause dans l'élaboration du devenir psychotique.

Par ailleurs, si un clinicien travaillant dans une clinique spécialisée voit superficiellement des centaines de cas, il est fort probable que son expérience le conduise à des conclusions contraires. J'ai vécu les deux situations et j'en suis arrivé à penser qu'il est excessivement dangereux de généraliser à l'ensemble des cas des conclusions élaborées à partir d'un seul pôle d'observation.

L. Q. : Et qu'en est-il de l'autisme?

P. D. : J'ai vu beaucoup d'enfants autistes et je considère qu'il s'agit d'un problème de manque de certaines fonctions à la base de la symbolisation et des interactions sociales. Je suis convaincu qu'il s'agit d'un problème qui a des causes neurologiques et qui se situe probablement dans la continuité de troubles graves du langage. À mes yeux, ce n'est pas une pathologie où le conflit pulsionnel occupe une place importante. J'ai connu beaucoup de parents d'enfants autistes qui m'ont paru admirables de patience et de générosité et qui faisaient preuve d'un engagement total avec un enfant qui, malheureusement, était souvent incapable de leur apporter des gratifications narcissiques. Il me semble aussi que dans l'autisme, il y a beaucoup de degrés à prendre en considération lors de l'orientation de ces enfants. Ce que je viens d'affirmer sur la place qu'occupe le conflit pulsionnel dans l'étiologie de l'autisme ne diminue en rien l'admiration que j'ai pour les travaux de Tustin, Meltzer, Hang, Ouzel et d'autres thérapeutiques d'orientation psychodynamique. Leurs descriptions du fonctionnement psychique des autistes sont certainement très utiles à ceux qui ont le courage de s'engager dans le traitement à long terme de ces enfants, ou qui veulent simplement comprendre leur monde mystérieux. 
Enfin il m'apparaît aussi essentiel de tenir compte des cas de pseudo-autisme qui ont été victimes de carences profondes dans les premiers mois de leur vie. J'ai examiné des enfants adoptés qui avaient, de toute évidence, subi des carences extrêmes dans les orphelinats des pays dont ils provenaient. Ces enfants déprimés utilisaient des mécanismes de défense de retrait, de non investissement qui donnaient un tableau assez semblable à celui de l'autisme. Dans leur cas, grâce au dévouement des parents adoptifs une certaine récupération a été possible, même si la plupart restent marqués par ces traumatismes primitifs.

L. Q. : Vous affirmez plus haut avoir rencontré des parents qui avaient sauvé leurs enfants d'une évolution vers une structure très pathologique. Iriez-vous jusqu'à dire qu'ils leur ont épargné la psychose?

P. D. : Je pensais aussi à l'autisme en disant cela. Je faisais allusion à des enfants particuliers, un peu bizarres qui auraient pu avoir une évolution désastreuse mais chez qui la catastrophe a vraiment été évitée par une attitude extraordinaire des parents. Les ressources des parents face à certains enfants malades n'ont pas fini de m'étonner. Par ailleurs, de faux espoirs ont été malheureusement donnés à certains parents lors de la proposition de thérapies excessivement intensives censées donner des résultats fabuleux, mais qui n'ont pas tenu leurs promesses. J'ai rencontré plusieurs parents qui se sont retrouvés dans cette situation et qui ont développé une grande culpabilité. Certains sont devenus très malheureux, d'autres se sont même séparés, car ils n’ont pu surmonter le traumatisme causé par la désillusion de leur croyance en une guérison miraculeuse.

L. Q. : Quelle place accordez-vous à la médication dans le traitement de la psychose?

P. D.: Chez l'adulte la médication peut jouer de toute évidence un rôle très important. Une des cures analytiques les plus fructueuses et intéressantes que j'ai menées, il y a une vingtaine d'années, se déroula avec un intellectuel, un universitaire qui avait des antécédents très importants de psychose maniaco-dépressive. À cette époque, j'avais demandé au docteur Grunberg, qui n'était pas un partisan de la psychanalyse, d'assumer le suivi médicamenteux de ce patient. Je pouvais voir dans les séances d'analyse s'il prenait ou non son médicament, du lithium, tellement sa pensée s'accélérait à un rythme incroyable quand il omettait de le prendre. De son côté, le docteur Grunberg se disait épaté des transformations qui se produisaient chez cet homme, très narcissique au début de la cure, qui se comportait en tyran avec les nombreuses femmes qu'il avait connues et qui, à la fin du traitement, était devenu un homme généreux, capable d'avoir une relation normale avec une compagne stable, tout en ayant une performance extraordinaire à son travail. La personnalité de cet homme a subi de profondes modifications, mais il continua néanmoins d'avoir besoin de son lithium.

Aujourd'hui, je pense que dans certains cas de psychose et de prépsychose rencontrés chez les enfants aussi bien que chez les adultes, les nouveaux médicaments sont nécessaires pour diminuer l'angoisse et favoriser corrélativement l'installation d'un processus thérapeutique. Bien sûr, il serait absurde de voir dans 
la médication une solution au problème, qui permettrait de faire l'économie du travail de psychothérapie. La pharmacologie a fait de très grands progrès et il est indéniable que les médicaments peuvent rendre de très grands services. Par ailleurs, il y a aussi en psychiatrie le danger d'évoluer vers une pratique déshumanisée, uniquement axée sur la disparition du symptôme, ce danger étant d'autant plus grand qu'il correspond aux demandes des instances gouvernementales. Il me semble que je n'ai pas à insister sur ce point tellement il est évident.

L. Q.: Abordons maintenant un autre de vos champs d'intérêt, l'enseignement. Favoriser la circulation des idées vous importe, comme en témoigne votre implication comme secrétaire scientifique pendant cinq ans à la Société psychanalytique de Montréal, mais j'ai l'impression que la transmission des connaissances aux générations montantes est une question qui vous tient encore plus à cœur. Depuis vingt-cinq ans, vous enseignez aux résidents en psychiatrie de l'Université de Montréal et coordonnez l'enseignement de la psychodynamique qui leur est dispensé. Comment concevez-vous l'avenir de la psychanalyse à l'université ?

P. D. : C'est une question difficile... Il est vrai que j'ai beaucoup travaillé pour que les futurs psychiatres puissent saisir qu'une deuxième dimension existe derrière les symptômes manifestes de leurs malades. Nous évoluons de plus en plus vers une psychiatrie symptomatique, tant en clinique infantile qu'en clinique adulte. Alors que nous savons que les mêmes symptômes peuvent être soutenus par des structures psychiques très différentes et que l'approche thérapeutique peut être complètement changée si on en tient compte; il me paraît essentiel dès lors de bien enseigner les éléments de base de la compréhension psychodynamique. Je dis compréhension psychodynamique parce qu'il serait utopique de prétendre enseigner la psychanalyse au niveau des études de résidence en psychiatrie. Par ailleurs, il me semble essentiel que les résidents puissent comprendre, saisir dans leur pratique qu'il y a différents types d'angoisse, différents types de mécanismes de défense, différents types de relations d'objet qui correspondent à différentes structures psychiques.

Je dois reconnaittre que la situation actuelle est difficile pour eux qui ont de plus en plus de connaissances à acquérir à divers niveaux. Ils doivent aussi apprendre de nouvelles approches psychothérapeutiques développées au cours des dernières années et le contexte médico-hospitalier, avec ses exigences décuplées de services, ne favorise pas le travail intensif auprès de certains patients. Par ailleurs ce qui est encourageant, c'est qu'en général les résidents apprécient beaucoup l'enseignement de la psychodynamique et que certains d'entre eux sont encore passionnés par cette dimension du fonctionnement psychique. Je dois dire que, lors d'affrontements avec la direction universitaire sur l'importance à donner à la compréhension psychodynamique, ils ont toujours été de mon côté. Dans une bataille récente sur la nécessité d'enseigner la psychothérapie d'orientation analytique à long terme, des résidents ont été les principaux défenseurs de la nécessité de la supervision par un thérapeute d'orientation analytique sur une période de deux ans. Et il commence à y avoir certains signes de relève, un certain nombre d'entre eux suivent 
eux-mêmes une psychanalyse, d'autres font des études post-doctorales sur des sujets psychanalytiques ou veulent aller faire des stages à l'étranger avec de grands psychanalystes.

Cela étant dit, je suis inquiet quand même, parce que les vents sont contraires, les psychiatres qui ont une formation analytique vieillissent et se retirent peu à peu de la scène, l'organisation des soins rend de plus en plus difficile l'approche psychodynamique. Malgré mon admiration face aux nouvelles découvertes de la biologie et le respect que j'ai pour ceux qui s'en servent dans la pratique, j'ai peur qu'on évolue vers une psychiatrie qui atteigne une grande efficacité dans le soulagement immédiat mais qui devienne parallèlement de plus en plus déshumanisée en ceci que le sens de l'individu, de la personne, du sujet soit en partie perdu.

L. Q. : Votre enseignement s'étaie sur une pratique clinique soutenue. Vous avez maintenu jusqu'à aujourd'hui, parallèlement à votre travail à demi temps auprès des enfants à l'hôpital Sainte-Justine, une pratique analytique importante en bureau privé. Quels sont les points d'appui théoriques qui guident votre action de clinicien, ceux que vous avez élevés au rang de références primordiales?

P. D. : Il me faut d'abord faire une distinction entre mon travail à l'hôpital avec les enfants et mon travail en bureau privé, presque exclusivement en psychanalyse. À l'hôpital où je suis en fin de carrière, j'avais choisi de travailler surtout avec les femmes enceintes et les jeunes mères. Après mon accident cérébro-vasculaire, qui m'a laissé avec une légère paralysie n'affectant pas heureusement le langage, on m'a donné un travail moins exigeant en clinique externe. J'aime toujours travailler avec les enfants, même à mon âge ; je suis encore émerveillé malgré la lourdeur de certains cas. Je me sers toujours de mes connaissances en psychodynamique pour répondre au niveau de la demande qui m'est faite. J'essaie de le faire d'une façon qui soit la plus simple et la plus concrète possible, et en utilisant tous les moyens dont nous disposons y compris la médication si elle est nécessaire. Je m'efforce de respecter la singularité de chaque enfant, de tenir compte de son vécu unique et de la profondeur de sa souffrance qui échappe souvent à une première vue. Je crois que je peux ainsi rendre encore de grands services.

En bureau privé, je pratique exclusivement la psychanalyse avec les adultes ; ma pratique est encore importante et j'y trouve toujours autant de bonheur et de passion; c'est le sel de ma vie.

Vous me demandiez quels sont les points d'appui théoriques qui guident mon action? Je répondrai de façon brève et condensée en me servant de ce qui m'a été le plus utile dans ma pratique. Je soulignerai d'abord la foi en la nécessité de respecter la dimension créatrice présente chez tous nos patients et qu'on doit rejoindre grâce, encore une fois, au holding interprétatif; il faut arriver à créer un espace de sécurité, de liberté, de jeu. Les moments charnières, les interprétations mutatives sont des événements peu fréquents dans une cure et ils ne se produisent que s'ils ont été longtemps préparés par l'instauration de cet espace de jeu et de sécurité. Qui plus est, la meilleure interprétation est, comme le disait Winnicott, celle qu'on amène le patient à se faire lui-même. 
Un autre point d'appui théorique essentiel réside dans la reconnaissance de la position dépressive telle que décrite et développée par Mélanie Klein, position qui implique la capacité de réparer les objets internes endommagés par la propre agressivité du sujet. La croyance en cette capacité de réparation dépend de la survie de l'objet externe -du thérapeute- aux attaques. C'est surtout Winnicott qui a attribué la capacité de croire en la réparation, à la survie de la mère aux attaques du petit enfant. Dans nos thérapies, lorsque surviennent beaucoup d'attaques contre le cadre et contre nous, il faut survivre sans rétaliation par l'interprétation ou les mouvements contre-transférentiels. Au pire de la tempête, il faut se dire qu'on est en fait au cœur du conflit et que l'essentiel de la partie est en train de se jouer. Survivre, savoir attendre, être excessivement patient, et demeurer créateur, sont les attitudes qui m'apparaissent fondamentales et sur lesquelles je ne saurais trop insister

Je pense aussi que c'est l'analyse de notre contre-transfert qui, en définitive, peut jouer sur la destinée d'une thérapie. Notre contre-transfert est inconscient, il nous échappe et c'est parfois en échangeant avec des collègues ou en réfléchissant dans l'après-coup sur certains événements qu'on peut le découvrir. C'est une dimension fondatrice de notre travail et même une longue expérience ne nous place pas à l'abri du jeu de notre inconscient qui nous échappe toujours. L'objectif du traitement est de permettre à un individu d'avoir accès à toute sa créativité. Certains symptômes peuvent persister, mais si on a libéré quelqu'un de certaines chaînes et qu'il peut devenir plus créateur, je pense qu'on lui a rendu le plus grand des services.

L. Q.: On lui a donné ce qu'il fallait pour poursuivre le travail par lui-même, en réalité.

P. D.: C'est cela, puisque ce travail se poursuivra pendant toute sa vie

L. Q. : En terminant, aimeriez-vous livrer un message aux thérapeutes d'aujourd'hui?

P. D. : C'est une tâche bien lourde que de livrer un message. Je suis encore jeune... et je ne veux pas avoir la sensation de faire un testament. J'ai déjà énoncé beaucoup d'observations et d'opinions qui découlent de ma pratique et de mon expérience clinique. J'ai voulu seulement témoigner de la grande chance que j'ai eue d'exercer un métier qui me passionne, un métier qui m'a coûté beaucoup d'efforts, m'a demandé beaucoup de travail et de réflexion et, parfois, m'a apporté des déceptions et des désillusions, mais un métier qui m'a surtout donné la chance d'explorer la nature humaine, de vivre des aventures intérieures troublantes mais fascinantes et d'aider, je devrais peut-être dire de réparer, ceux qui n'ont pas eu la chance d'échapper à la catastrophe ou de simplement vivre pleinement leur vie de façon créatrice. La psychanalyse avec les adultes et les enfants a été pour moi le «sel de la terre», le vent de la mer et, avec ceux que j'aime, le cœur de ma vie. Je souhaite à ceux qui prennent la relève de vivre intensément et de ne jamais perdre espoir dans les capacités de guérison de ceux qu'il essaieront d'aider.

L. Q. : Merci beaucoup, $D^{r}$ Drapeau, de cet entretien. 


\section{Liste des publications de Pierre Drapeau}

Gauthier, Y., Fortin, C., Drapeau, P., Breton, J. J., Quintal, L., Gosselin, J., Weisnagel, J., 1973, Asthme infantile: allergie et psychopathologie, L'Union médicale du Canada, vol. 102, n $11,2342-2346$.

Gauthier, Y., Fortin, C., Drapeau, P., Breton, J. J., Gosselin, J., Quintal, L., Weisnagel, J., 1976, L'asthme chez le très jeune enfant (14-30 mois) : caractéristiques allergiques et psychologiques, Psychiatrie de l'enfant, vol. 19, $\mathrm{n}^{\circ} 1$, 3-146.

Drapeau, P.,1976, Réflexions sur la maladie à partir de l'étude de dessins d'enfants, Cahiers de pédopsychiatrie de l'Hôpital Sainte-Justine, $\mathrm{n}^{\circ}$ 5, 75-96.

Gauthier, Y., Fortin, C., Drapeau, P., Breton, J.J., Quintal, L., Gosselin, J., Weisnagel, J., Lamarre, A., 1977, L'asthme chez l'enfant de 4 à 6 ans, L'Union médicale du Canada, vol. 106, nº 7, 974-984.

Drapeau, P., 1977, La psychanalyse des enfants, Cahier spécial René Diatkine. Cahiers de pédopsychiatrie de l'Hôpital Sainte-Justine, $\mathrm{n}^{\circ}$ 13, 11-28.

Drapeau, P., 1977, Au-delà de la quantité, quelques réflexions sur la sectorisation, Cahiers de pédopsychiatrie de l'Hôpital Sainte-Justine, $\mathrm{n}^{\circ}$ 8, 5-8.

Drapeau, P., 1980, Asthme, allergie, développement psychologique chez le jeune enfant, Psychologie médicale, vol.12, n ${ }^{\circ} 5, .1023-1038$.

Drapeau, P., 1991, Jeu, créativité et œuvre d'art, Prisme, vol.2, no2, 155-166.

Drapeau, P., 1993, Écho d'une très lointaine enfance, L'inconscient, Ed. L'inconscient, Montréal, 85-107.

Drapeau, P., 2000, L'infantile et les idéaux, Revue française de psychanalyse, vol.5, 1661-1669.

Drapeau, P., 2002, From Freud to Winnicott. The Illusive Child, Karnac The Squiggle Fondation, 15-44.

\section{Liste des conférences et communications de Pierre Drapeau}

Drapeau, P., 1978, Potentiel allergique et développement psychologique de l'enfant, Congrès de médecine psychosomatique, Auxerre.

Drapeau, P., 1979, Mélanie Klein et la vie fantasmatique des enfants, Montpellier.

Drapeau, P., 1982, Voyage au pays des fantasmes originaires. Psychanalyse d'un enfant psychotique, Société psychanalytique de Montréal, Montréal.

Drapeau, P., 1982,. L'enfant soleil. Réflexions sur le processus thérapeutique, Congrès des psychanalystes de langue française, Montréal.

Drapeau, P., 1984, Les contes de la folie. Suite de la psychanalyse d'un enfant psychotique, Société psychanalytique de Montréal, Montréal.

Drapeau, P.,1984, L'identification projective, Société psychanalytique de Montréal, Montréal.

Drapeau, P., 1987, Commentaire sur la présentation de Takeo Doi, Branche québécoise de la société canadienne de psychanalyse, Montréal.

Drapeau, P., 1987, Commentaire principal sur la présentation de Steven Luria Ablon, Congrès de l'association psychanalytique internationale, Montréal.

Drapeau, P., 1989, Commentaire principal sur la présentation de Scott Dowling, Congrès de l'association psychanalytique internationale, Rome.

Drapeau, P., 1991, Les théories sexuelles infantiles, Colloque de l'association des psychothérapeutes psychanalytiques du Québec, Montréal.

Drapeau, P., 1995, Situer Winnicott, Société psychanalytique de Montréal, Montréal.

Drapeau, P., 1995, Hans, mon frère Hans, ne vois-tu rien venir? L'enfant dans la psychanalyse, conférence publique annuelle de la société psychanalytique de Montréal, Montréal. 
Filigrane, printemps 2006

Drapeau, P., 1997, Des théories et des dragons, première partie: Réflexions sur les théories sexuelles infantiles et la vie fantasmatique des enfants, Société psychanalytique de Montréal, Montréal.

Drapeau, P., 1997, Des théories et des dragons, deuxième partie : Le ventre des dragons, Société psychanalytique de Montréal, Montréal.

Drapeau, P., 1999, Winnicott. L'être et la transitionalité, Journée d'étude de l'association des psychothérapeutes psychanalytiques du Québec, Montréal.

Drapeau, P., 1999, La vie fantasmatique des enfants, Association des psychothérapeutes psychanalytiques du Québec, Montréal.

Drapeau, P., 2000, Le fantasme chez l'enfant, Société canadienne de psychanalyse, section psychanalyse de l'enfant, Montréal.

Drapeau, P., 2000, L'infantile et les idéaux, Congrès des psychanalystes de langue française, Montréal.

Drapeau, P., Pépin, H. P., 2000, L'approche par les ateliers thérapeutiques, Congrès mondial de la WAIM, Montréal.

Drapeau, P., 2001, L'archä̈que, Colloque de l'association des psychothérapeutes psychanalytiques du Québec, Montréal.

Drapeau, P., 2001, De Freud à Winnicott. Rencontre des enfants mythiques, Société psychanalytique de Montréal, Montréal.

Drapeau, P., Mauger, J., 2003, Les maîtres, Société psychanalytique de Montréal, Montréal.

Drapeau, P., 2003, The Mythical Children, Branche québécoise de la société canadienne de psychanalyse, Montréal.

Drapeau, P., 2003, Winnicott, Journée d'étude de la société psychanalytique d'Ottawa, Ottawa. 